\title{
PROXIMATE ANALYSIS OF FORAGE SORGHUM CULTIVARS WITH DIFFERENT DOSES OF NITROGEN AND SEED RATE
}

\author{
Ahmad SHER ${ }^{*}$, Muhammad ANSAR ${ }^{2}$, Muhammad IJAZ ${ }^{1}$ and Abdul SATTAR ${ }^{1}$ \\ ${ }^{1}$ Bahauddin Zakariya University, College of Agriculture, Bahadur Campus Layyah, PAKISTAN \\ ${ }^{2}$ PMAS-Arid Agriculture University Rawalpindi, Department of Agronomy, PAKISTAN \\ *Corresponding author: ahmad.sher@bzu.edu.pk
}

Received: 18.08.2015

\begin{abstract}
In this study, change in proximate composition in forage sorghum cultivars as influenced by nitrogen $(\mathrm{N})$ fertilization and seed rate harvested at different growth stages was planned through field experiments during consecutive years. Three forage sorghum cultivars viz., JS-2002, JS-263 and Chakwal sorghum were sown under three $N$ levels $\left(0,60\right.$ and $\left.120 \mathrm{~kg} \mathrm{ha}^{-1}\right)$ by using three seed rates i.e. 75, 100 and $125 \mathrm{~kg} \mathrm{ha}^{-1}$. Results showed that all individual effect of treatments had a variable influence on quality of sorghum cultivars. JS2002 had higher crude protein content (CP), crude protein yield (CPY), crude fibre (CF), crude fibre yield (CFY), ether extractable fats (EE), total ash content (TA) and organic matter (OM) whilst JS-263 produced greater nitrogen free extract (NFE) than other cultivars. Increasing $N$ rates $\left(0\right.$ to $\left.120 \mathrm{~kg} \mathrm{ha}^{-1}\right)$ raised all above mention bio-chemical attributes but not NFE. Similarly, increased seed rate enhanced accumulation of CP, CF, EE, TA, CPY and CFY while caused reduction of OM and NFE. Genotypic variations may have influenced the accumulations of these traits however, differences were not significant. In conclusion, sorghum cultivar JS-2002 seeded at $75 \mathrm{~kg} \mathrm{ha}^{-1}$ with $120 \mathrm{~kg} \mathrm{~N} \mathrm{ha}^{-1}$ application produced better quality forage under subtropical conditions
\end{abstract}

Keywords: Forage sorghum cultivars, seed rate, $\mathrm{N}$ levels, proximate quality, growth stages, sub-tropical conditions

List of Abbreviations: a.s.l, Above sea level; ANOVA, analysis of variance; C, cultivar; CP, crude protein; $\mathrm{CF}$, crude fibre; EE, ether extractable fats, DAE, days after emergence; K, potassium; N, nitrogen; NFE, nitrogen free extract; OM, organic matter; P, phosphorus; SNK, Scott-Newman-Keuls; TA, total ash content; Y, year.

\section{INTRODUCTION}

Livestock is an integral part of farming system in Pakistan and its population going to be increasing day by day. This increase in change between 1996 to 2006 is about $29 \%$ but area under fodder production decreases about $8.07 \%$ between this era (GOP, 2007). There is severe shortage of forage during winter months because of shortage of rainfall and about $60 \%$ of livestock feed on wheat straw, dry stalks of maize, sorghum and millet during winter months. Farmer grows wheat and brassica as a green fodder crops for winter feeding to their livestock (Kumar and Thakur, 2006).

Sorghum is an important summer season crop sown both for fodder and grain purposes. Average fodder yield at present under local conditions is less than the potential of 50-100 tons per hectare. Sorghum can resist drought and hot weather and can be successfully grown on all types of soil except water logged and saline (Chaudhry et al., 2006). Forage shortage during the scarcity period can be reduced by the introducing the high yielding cultivars
(Chohan et al., 2006). Different sorghum cultivars vary in fodder yield as well as quality of fodder (Sarfraz et al., 2012; Bilal et al., 2001; Chohan et al., 2006).

The introduction of high yielding crop varieties is the most suitable option to fulfill forage shortage (Bilal et al., 2001). Significant differences in the results have been observed among the different sorghum cultivars for quality attributes (Ashraf et al., 1995; Sarfraz et al., 2012).

Nitrogen $(\mathrm{N})$ fertilizer plays a key role in increasing sorghum forage yield with good nutrition value especially in rainfed regions of the world. It is very important agricultural utility in various developed and developing countries. The use of heavy doses of $\mathrm{N}$ to achieve increased fodder has become a custom as it not only increases the yield but protein also (Mohammed and Hamed, 1988). With the increased use of $\mathrm{N}$, crude fibre contents are reduced (Patel et al., 1994). Increase in $\mathrm{N}$ levels also increases the protein and digestibility of dry matter. This practice may change the canopy architecture by increased size of foliage. High concentration of $\mathrm{N}$ 
results in succulent growth and dense canopy of plants. High usage of $\mathrm{N}$ may increase the vegetative growth and maturity duration of the plants and has affected the quality of the sorghum forage (Rana et al., 1990).

Population density and $\mathrm{N}$ application have great influence on quality of sorghum forage. The input of any physical resource below the optimum level, change the growth of plant and yield (Clipson and Edwards, 1994).

In the light of the above mentioned facts, an investigation was undertaken to study the changes in proximate composition in forage sorghum cultivars as influenced by nitrogen fertilization and seed rate.

\section{MATERIALS AND METHODS}

Site description

Field experiment was carried out during the summer 2008 and 2009 at National Agricultural Research Centre (NARC; 334' $\mathrm{N}$; 7304' E; $518 \mathrm{~m}$ a.s.l), Islamabad. Figure 1 shows meteorological data of the experimental area during the growing season, with monthly average temperature and monthly total rainfall. Soil of the experimental area is clay-loam with neutral $\mathrm{pH}$. Total $\mathrm{N}$ and organic matter contents were low, particularly in the layers to below given depth. There was poor phosphorus and potassium status in the soil.

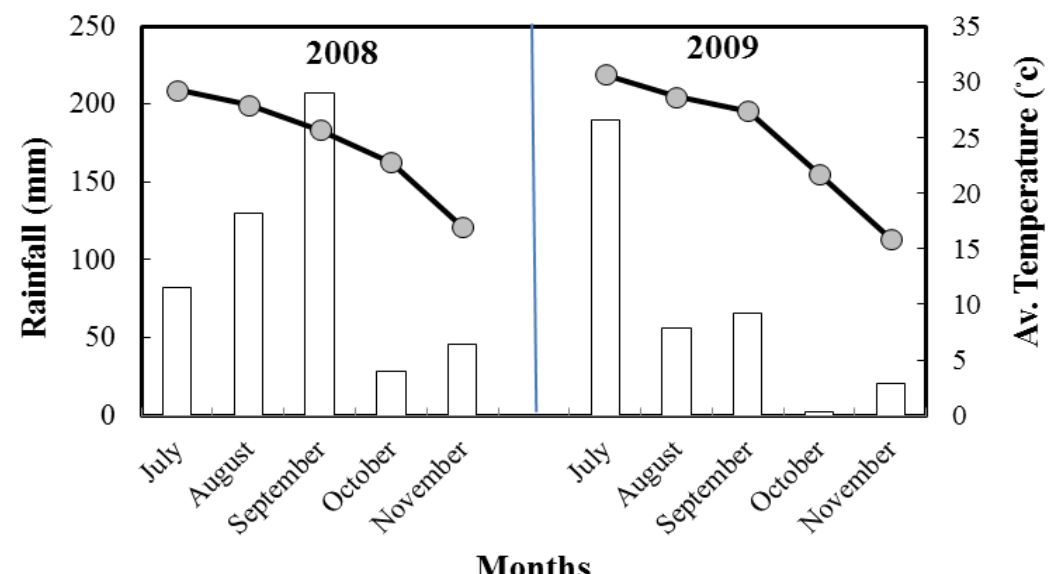

Figure 1. Rainfall, av. temperature and relative humidity recorded during crop growth period

\section{Plant material and experimental design}

Three forage sorghum cultivars: two cultivars i.e. JS2002 and Chakwal sorghum were approved by Punjab Seed Council in collaboration with Seed Certification \& Registration Department and one landrace (JS-263). The cultivar JS-2002 is the late variety which remains green for a longer period with small/compact panicle and yellow grain for irrigated areas and is developed by Fodder Research Institute, Sargodha. Chakwal Sorghum is a medium cycle cultivar for rainfed areas, resistant to drought with loose panicle and whitish grain and is being developed by Barani Agricultural Research Institute, Chakwal. JS-263 seed was collected from farmers of Rawalpindi surrounding rainfed area; is a medium-high genotype, semi-resistant to drought, with loose panicle, bold and whitish grain. These cultivars were grown each with 75,100 and $125 \mathrm{~kg} \mathrm{ha}^{-1}$ seeding rates, and under 0 , 60 and $120 \mathrm{~kg} \mathrm{ha}^{-1}$ nitrogen (N) levels. Sorghum cultivars, seed rates and $\mathrm{N}$ rates were allocated in main plots, subplots and sub-sub plots, respectively. This experiment comprised of 27 treatment combinations under randomized completely block design arranged in splitsplit plots with three replications.

\section{Crop husbandry}

Crop was sown on $20^{\text {th }}$ August, 2008 and $1^{\text {st }}$ August, 2009 with same area of treatment plots size was $4 \mathrm{~m} \times 1.2$ $\mathrm{m}$ during two years, respectively. Planting of crop was done by maintaining $30 \mathrm{~cm}$ of row to row distance with hand drill. At the time of seed bed preparation, applied 30 $\mathrm{kg} \mathrm{ha}^{-1}$ of phosphorus as triple super phosphate. The $\mathrm{N}$ was applied in the form of urea through broadcasting as per treatment. All other cultural practices were kept uniform as recommended for sorghum forage crop during both seasons.

\section{Forage quality analysis and calculations}

The proximate composition viz., crude protein, CF, EE, TA, NFE and OM were determined for all samples. Nitrogen concentrations were determined by the Kjeldahl procedure and crude protein concentration was calculated by the formula of $\mathrm{N}$ concentration $\times 6.25$ (AOAC, 2002). Crude protein and fibre yield is estimated to multiply crude protein percentage and crude fibre with dry matter yield, respectively and then dividing it by 100 . 
The data collected were subjected to analysis of variance using the CoStat 6.3 software (CoHort Software, Monterey, CA, USA) to determine significance of the tested factors (cultivar, seed rate and $\mathrm{N}$ level) with sorghum traits. Means of significant effects were separated using the test at $5 \%$ level of significance.

\section{RESULTS}

\section{Crude protein}

Amongst cultivars, JS-2002 gave maximum crude protein (CP) than JS-263 (Table 1). JS-2002 produced CP 43.8 and $31.3 \%$ at pre-booting, 36 and $31.5 \%$ at booting, 26.1 and $25.9 \%$ at $50 \%$ heading stage in 2008 and 2009 , respectively; which is higher than JS-263. Increase in seed rate gradually decreased crude protein. Lower seed rate produced highest $\mathrm{CP}$ at pre-booting, booting and $50 \%$ heading stage during both the years. Nitrogen rate is linearly related with the value of crude protein. Higher dose of $N\left(120 \mathrm{~kg} \mathrm{ha}^{-1}\right)$ resulted maximum $\mathrm{CP}$, while minimum was found from zero $\mathrm{N}$ application at three successive growth stages during both years of study. Almost similar trend was found during both years; however, overall $\mathrm{CP}$ in all the treatments was better during 2009 than 2008.

\section{Crude protein yield}

Statistically analysis of crude protein yield (CPY) revealed that means comparison of sorghum cultivars indicate that JS-2002 produced significantly higher CPY (t $\mathrm{ha}^{-1}$ ) than JS-263 and Chakwal sorghum (Table 2). Increasing seed rates decreased the CPY $\left(\mathrm{t} \mathrm{ha}^{-1}\right)$. Higher CPY $\left(\mathrm{t} \mathrm{ha}^{-1}\right)$ was recorded at lower seed rate $\left(75 \mathrm{~kg} \mathrm{ha}^{-1}\right)$ than at higher seed rate at three successive growth stages in the two consecutive years. Regardless of seed rate, $\mathrm{N}$ application rate showed direct relation with CPY $\left(\mathrm{t} \mathrm{ha}^{-1}\right)$. Maximum CPY ( $\left.\mathrm{t} \mathrm{ha}^{-1}\right)$ at pre-booting, booting and $50 \%$ heading stage was recorded at higher rate $120 \mathrm{~kg} \mathrm{~N} \mathrm{ha}^{-1}$ during both years.

\section{Crude fibre}

Statistical analysis of data revealed that individual effect of sorghum cultivars, seed rate and $\mathrm{N}$ application significantly $(\leq 0.05)$ affected the crude fibre (CF). JS2002 produced significantly higher contents of crude fibre at all stage (Table 1). Contrarily, JS-263 contained minimum $\mathrm{CF}$ at all three succeeding growth stages during both years of study. Seed rate inversely affect $\mathrm{CF}$ values. Maximum CF was recorded at lower seed rate $\left(75 \mathrm{~kg} \mathrm{ha}^{-1}\right)$ than higher rate $\left(125 \mathrm{~kg} \mathrm{ha}^{-1}\right)$. Increasing nitrogen level increased the $\mathrm{CF}$. The highest $\mathrm{CF}$ at pre-booting, booting and $50 \%$ heading stage was recorded with higher dose (120 kg N ha ${ }^{-1}$ ) during two years.

\section{Crude fibre yield}

Data revealed that individual effect of sorghum cultivars, seed rate and $\mathrm{N}$ application significantly $(\leq 0.05)$ affected the crude fibre yield (CFY). JS-2002 produced significantly higher crude fibre yield at all stages (Table 2). Seed rate inversely affect CFY values. Maximum CFY was recorded at lower seed rate $\left(75 \mathrm{~kg} \mathrm{ha}^{-1}\right)$ than higher rate $\left(125 \mathrm{~kg} \mathrm{ha}^{-1}\right)$. Increasing $\mathrm{N}$ level increased the CFY. The highest CFY at pre-booting, booting and $50 \%$ heading stage was recorded with higher dose $\left(120 \mathrm{~kg} \mathrm{~N} \mathrm{ha}^{-1}\right)$ during two years.

\section{Ether extractable fats}

Among the sorghum cultivars, maximum ether extracts (EE) at pre-booting, booting and 50\% heading stage were recorded in JS-2002, whereas minimum EE was observed in JS-263 at three successive growth stages during two consecutive years (Table 3). Increasing seed rates increased the EE. Maximum EE were observed with seed rate of $75 \mathrm{~kg} \mathrm{ha}^{-1}$ during both the years. Increasing $\mathrm{N}$ levels increased the EE concentration. The highest EE was recorded with application of $120 \mathrm{~kg} \mathrm{~N} \mathrm{ha}^{-1}$.

\section{Total ash content}

Means comparison of sorghum cultivars indicate that JS-2002 produced significantly more total ash content (TA) than JS-263 and chakwal sorghum (Table 3). Increasing seeding rates increased the TA. Maximum TA was recorded at higher rate $\left(125 \mathrm{~kg} \mathrm{ha}^{-1}\right)$ at three successive growth stages in the two consecutive years. Regardless of seed rate, $\mathrm{N}$ application rate showed direct relation with TA. Maximum TA at pre-booting, booting and $50 \%$ heading stage was recorded at higher rate $120 \mathrm{~kg}$ $\mathrm{N} \mathrm{ha}^{-1}$. In contrast, minimum TA was observed in control plots $\left(0 \mathrm{~kg} \mathrm{~N} \mathrm{ha}^{-1}\right)$ during both years.

\section{Nitrogen free extract}

Amongst cultivars, JS-263 contained higher nitrogen free extract (NFE) as compared to JS-2002 (Table 4). Increased seed rates resulted in progressive increase of NFE. Maximum NFE was recorded at seed rate $125 \mathrm{~kg}^{-}$ ${ }^{1}$ than 100 and $75 \mathrm{~kg} \mathrm{ha}^{-1}$ seed rate during 2008 and 2009 at respective growth stages. The $\mathrm{N}$ rate showed inverse relationship with NFE. Highest NFE was recorded from treatment without $\mathrm{N}$ application when compared with high dose $\left(120 \mathrm{~kg} \mathrm{~N} \mathrm{ha}^{-1}\right)$ at three successive stages during two consecutive years.

\section{Organic matter}

Statistical analysis of data showed that JS-2002 produced maximum organic matter (OM) value compared to JS-263 at pre-booting stage during 2008 and 2009 respectively, while booting and $50 \%$ heading stage during 2009 only (Table 4). Higher seed rates gave higher OM. Maximum OM was recorded at higher seed rate $(125 \mathrm{~kg}$ $\mathrm{ha}^{-1}$ ) in comparison to lower rates (100 and $\left.75 \mathrm{~kg} \mathrm{ha}^{-1}\right)$. Likewise, $\mathrm{N}$ application enhanced organic matter at prebooting, booting and $50 \%$ heading stage during both years with $120 \mathrm{~kg} \mathrm{~N} \mathrm{ha}^{-1}$. 
Table 1. Interactive effect of Cultivar, Seed rate and Nitrogen application of sorghum forage at different growth stages during 2008 and 2009

\begin{tabular}{|c|c|c|c|c|c|c|c|c|c|c|c|c|}
\hline \multirow{3}{*}{ Treatments } & \multicolumn{6}{|c|}{ Crude protein (\%) } & \multicolumn{6}{|c|}{ Crude fibre (\%) } \\
\hline & \multicolumn{2}{|c|}{ Pre-booting } & \multicolumn{2}{|c|}{ Booting } & \multicolumn{2}{|c|}{$50 \%$ heading } & \multicolumn{2}{|c|}{ Pre-booting } & \multicolumn{2}{|c|}{ Booting } & \multicolumn{2}{|c|}{$50 \%$ heading } \\
\hline & 2008 & 2009 & 2008 & 2009 & 2008 & 2009 & 2008 & 2009 & 2008 & 2009 & 2008 & 2009 \\
\hline \multicolumn{13}{|l|}{ Cultivar (C) } \\
\hline JS-2002 & $11.5 \mathrm{a}$ & $13.0 \mathrm{a}$ & $10.2 \mathrm{a}$ & $12.1 \mathrm{a}$ & $8.7 \mathrm{a}$ & $10.7 \mathrm{a}$ & $41.9 \mathrm{a}$ & $39.4 \mathrm{a}$ & $31.3 \mathrm{a}$ & $29.2 \mathrm{a}$ & $27.8 \mathrm{a}$ & $25.9 \mathrm{a}$ \\
\hline $\begin{array}{l}\text { Chakwal } \\
\text { sorghum }\end{array}$ & $8.9 \mathrm{~b}$ & $10.9 \mathrm{~b}$ & $8.0 \mathrm{~b}$ & $9.9 \mathrm{~b}$ & $7.5 \mathrm{~b}$ & $9.2 \mathrm{~b}$ & $39.1 \mathrm{~b}$ & $37.3 \mathrm{~b}$ & $29.2 \mathrm{~b}$ & $27.0 \mathrm{~b}$ & $27.0 \mathrm{a}$ & $25.0 \mathrm{~b}$ \\
\hline JS-263 & $8.0 \mathrm{c}$ & $9.9 \mathrm{c}$ & $7.5 \mathrm{c}$ & $9.2 \mathrm{c}$ & $6.9 \mathrm{c}$ & $8.5 \mathrm{c}$ & $35.4 \mathrm{c}$ & $33.6 \mathrm{c}$ & $25.8 \mathrm{c}$ & $23.6 \mathrm{c}$ & $25.1 \mathrm{~b}$ & $23.1 \mathrm{c}$ \\
\hline LSD & 0.528 & 0.343 & 0.369 & 0.400 & 0.331 & 0.362 & 0.770 & 0.622 & 0.919 & 1.309 & 0.792 & 0.753 \\
\hline \multicolumn{13}{|c|}{ Seed rate $(S)\left(\mathrm{kg} \mathrm{ha}^{-1}\right)$} \\
\hline 75 & $10.4 \mathrm{a}$ & $12.4 \mathrm{a}$ & $9.5 \mathrm{a}$ & $11.6 \mathrm{a}$ & $8.6 \mathrm{a}$ & $10.5 \mathrm{a}$ & $41.1 \mathrm{a}$ & $38.6 \mathrm{a}$ & $30.6 \mathrm{a}$ & $28.2 \mathrm{a}$ & $28.6 \mathrm{a}$ & $26.5 \mathrm{a}$ \\
\hline 100 & $9.7 \mathrm{~b}$ & $11.4 \mathrm{~b}$ & $8.6 \mathrm{~b}$ & $10.5 \mathrm{~b}$ & $7.7 \mathrm{~b}$ & $9.4 \mathrm{~b}$ & $38.8 \mathrm{~b}$ & $36.8 \mathrm{~b}$ & $28.9 \mathrm{~b}$ & $26.7 \mathrm{~b}$ & $26.6 \mathrm{~b}$ & $24.6 \mathrm{~b}$ \\
\hline 125 & $8.3 \mathrm{c}$ & $10.0 \mathrm{c}$ & $7.5 \mathrm{c}$ & $9.2 \mathrm{c}$ & $6.9 \mathrm{c}$ & $8.5 \mathrm{c}$ & $36.4 \mathrm{c}$ & $35.0 \mathrm{c}$ & $26.8 \mathrm{c}$ & $24.9 \mathrm{c}$ & $24.6 \mathrm{c}$ & $22.8 \mathrm{c}$ \\
\hline LSD & 0.528 & 0.343 & 0.369 & 0.400 & 0.331 & 0.362 & 0.770 & 0.622 & 0.919 & 1.309 & 0.792 & 0.753 \\
\hline \multicolumn{13}{|c|}{ Nitrogen level (N) $\left(\mathrm{kg} \mathrm{ha}^{-1}\right)$} \\
\hline 0 & $8.5 \mathrm{c}$ & $10.0 \mathrm{c}$ & $7.5 \mathrm{c}$ & $9.1 \mathrm{c}$ & $6.4 \mathrm{c}$ & $8.2 \mathrm{c}$ & $36.4 \mathrm{c}$ & $34.4 \mathrm{c}$ & $27.3 \mathrm{c}$ & $25.1 \mathrm{c}$ & $25.1 \mathrm{c}$ & $23.5 \mathrm{c}$ \\
\hline 60 & $9.6 \mathrm{~b}$ & $11.4 \mathrm{~b}$ & $8.5 \mathrm{~b}$ & $10.3 \mathrm{~b}$ & $7.7 \mathrm{~b}$ & $9.4 \mathrm{~b}$ & $28.8 \mathrm{~b}$ & $37.0 \mathrm{~b}$ & $28.8 \mathrm{~b}$ & $26.6 \mathrm{~b}$ & $26.5 \mathrm{~b}$ & $24.5 \mathrm{~b}$ \\
\hline 120 & $10.2 \mathrm{a}$ & $12.5 \mathrm{a}$ & $9.6 \mathrm{a}$ & $11.8 \mathrm{a}$ & $9.0 \mathrm{a}$ & $10.7 \mathrm{a}$ & $41.1 \mathrm{a}$ & $38.9 \mathrm{a}$ & $30.2 \mathrm{a}$ & $28.1 \mathrm{a}$ & $28.3 \mathrm{a}$ & $26.0 \mathrm{a}$ \\
\hline LSD & 0.528 & 0.343 & 0.369 & 0.400 & 0.331 & 0.362 & 0.770 & 0.622 & 0.919 & 1.309 & 0.792 & 0.753 \\
\hline $\mathbf{C} \times \mathbf{S}$ & $<0.006^{*}$ & $<0.001 * *$ & $0.052 \mathrm{~ns}$ & $<0.001 * *$ & $0.215 \mathrm{~ns}$ & $0.139 \mathrm{~ns}$ & $0.773 \mathrm{~ns}$ & $0.106 \mathrm{~ns}$ & $0.476 \mathrm{~ns}$ & $0.089 \mathrm{~ns}$ & $0.207 \mathrm{~ns}$ & $0.789 \mathrm{~ns}$ \\
\hline $\mathbf{C} \times \mathbf{N}$ & $0.778 \mathrm{~ns}$ & $<0.043 *$ & $0.252 \mathrm{~ns}$ & $0.100 \mathrm{~ns}$ & $0.708 \mathrm{~ns}$ & $0.950 \mathrm{~ns}$ & $0.924 \mathrm{~ns}$ & $<0.027 *$ & $0.721 \mathrm{~ns}$ & $0.498 \mathrm{~ns}$ & $0.884 \mathrm{~ns}$ & $0.349 \mathrm{~ns}$ \\
\hline $\mathbf{S} \times \mathbf{N}$ & $0.893 \mathrm{~ns}$ & $<0.029 *$ & $0.754 \mathrm{~ns}$ & $0.706 \mathrm{~ns}$ & $0.586 \mathrm{~ns}$ & $0.177 \mathrm{~ns}$ & $0.969 \mathrm{~ns}$ & $0.196 \mathrm{~ns}$ & $0.878 \mathrm{~ns}$ & $0.949 \mathrm{~ns}$ & $0.737 \mathrm{~ns}$ & $0.091 \mathrm{~ns}$ \\
\hline $\mathbf{C} \times \mathbf{S} \times \mathbf{N}$ & $0.982 \mathrm{~ns}$ & $0.338 \mathrm{~ns}$ & $0.859 \mathrm{~ns}$ & $0.600 \mathrm{~ns}$ & $0.357 \mathrm{~ns}$ & $0.493 \mathrm{~ns}$ & $0.717 \mathrm{~ns}$ & $<0.005^{*}$ & $0.973 \mathrm{~ns}$ & $0.531 \mathrm{~ns}$ & $0.976 \mathrm{~ns}$ & $0.863 \mathrm{~ns}$ \\
\hline C.V. (\%) & 10.1 & 5.5 & 7.8 & 7.0 & 7.8 & 6.9 & 3.6 & 3.1 & 5.8 & 8.9 & 5.4 & 5.3 \\
\hline
\end{tabular}

Different letters indicate statistically significant-difference among the values in each column and individual factors (SNK test; $P \leq 0.05$ ) 
Table 2. Interactive effect of Cultivar, Seed rate and Nitrogen application of sorghum forage at different growth stages during 2008 and 2009

\begin{tabular}{|c|c|c|c|c|c|c|c|c|c|c|c|c|}
\hline \multirow{3}{*}{ Treatments } & \multicolumn{6}{|c|}{ Crude protein yield $\left(\mathrm{t} \mathrm{ha}^{-1}\right)$} & \multicolumn{6}{|c|}{ Crude fibre yield (t ha-1) } \\
\hline & \multicolumn{2}{|c|}{ Pre-booting } & \multicolumn{2}{|c|}{ Booting } & \multicolumn{2}{|c|}{$50 \%$ heading } & \multicolumn{2}{|c|}{ Pre-booting } & \multicolumn{2}{|c|}{ Booting } & \multicolumn{2}{|c|}{$50 \%$ heading } \\
\hline & 2008 & 2009 & 2008 & 2009 & 2008 & 2009 & 2008 & 2009 & 2008 & 2009 & 2008 & 2009 \\
\hline \multicolumn{13}{|l|}{ Cultivar (C) } \\
\hline JS-2002 & $1.42 \mathrm{a}$ & $1.02 \mathrm{a}$ & $1.76 \mathrm{a}$ & $1.13 \mathrm{a}$ & $2.09 \mathrm{a}$ & $1.97 \mathrm{a}$ & $5.14 \mathrm{a}$ & $3.06 \mathrm{a}$ & $5.34 \mathrm{a}$ & $2.66 \mathrm{a}$ & $6.62 \mathrm{a}$ & $4.75 \mathrm{a}$ \\
\hline $\begin{array}{l}\text { Chakwal } \\
\text { sorghum }\end{array}$ & $0.75 \mathrm{~b}$ & $0.58 \mathrm{~b}$ & $1.13 \mathrm{~b}$ & $0.78 \mathrm{~b}$ & $1.32 \mathrm{~b}$ & $1.05 \mathrm{~b}$ & $3.24 \mathrm{~b}$ & $1.95 \mathrm{~b}$ & $4.05 \mathrm{~b}$ & $2.09 \mathrm{~b}$ & $4.70 \mathrm{~b}$ & $2.79 \mathrm{~b}$ \\
\hline JS-263 & $0.44 \mathrm{c}$ & $0.32 \mathrm{c}$ & $0.77 \mathrm{c}$ & $0.41 \mathrm{c}$ & $0.91 \mathrm{c}$ & $0.65 \mathrm{c}$ & $1.93 \mathrm{c}$ & $1.07 \mathrm{c}$ & $2.62 \mathrm{c}$ & $1.04 \mathrm{c}$ & $3.24 \mathrm{c}$ & $1.74 \mathrm{c}$ \\
\hline LSD & 0.187 & 0.084 & 0.299 & 0.111 & 0.303 & 0.345 & 0.729 & 0.236 & 0.876 & 0.268 & 0.950 & 0.749 \\
\hline \multicolumn{13}{|c|}{ Seed rate $(S)\left(\mathrm{kg} \mathrm{ha}^{-1}\right)$} \\
\hline 75 & $1.11 \mathrm{a}$ & $0.88 \mathrm{a}$ & $1.62 \mathrm{a}$ & $1.03 \mathrm{a}$ & $1.62 \mathrm{a}$ & $1.45 \mathrm{a}$ & $4.29 \mathrm{a}$ & $2.66 \mathrm{a}$ & $5.06 \mathrm{a}$ & $2.47 \mathrm{a}$ & $5.73 \mathrm{a}$ & $3.79 \mathrm{a}$ \\
\hline 100 & $0.88 \mathrm{~b}$ & $0.63 \mathrm{~b}$ & $1.20 \mathrm{~b}$ & $0.74 \mathrm{~b}$ & $1.39 \mathrm{~b}$ & $1.18 \mathrm{~b}$ & $3.39 \mathrm{~b}$ & $1.99 \mathrm{~b}$ & $3.92 \mathrm{~b}$ & $1.84 \mathrm{~b}$ & $4.66 \mathrm{~b}$ & $2.95 \mathrm{~b}$ \\
\hline 125 & $0.61 \mathrm{c}$ & $0.42 \mathrm{c}$ & $0.86 \mathrm{c}$ & $0.55 \mathrm{c}$ & $1.13 \mathrm{c}$ & $1.03 \mathrm{c}$ & $2.62 \mathrm{c}$ & $1.42 \mathrm{c}$ & $3.02 \mathrm{c}$ & $1.47 \mathrm{c}$ & $4.17 \mathrm{c}$ & $2.53 \mathrm{c}$ \\
\hline LSD & 0.092 & 0.056 & 0.106 & 0.077 & 0.084 & 0.096 & 0.299 & 0.124 & 0.245 & 0.175 & 0.300 & 0.279 \\
\hline \multicolumn{13}{|c|}{ Nitrogen level (N) $\left(\mathrm{kg} \mathrm{ha}^{-1}\right)$} \\
\hline 0 & $0.59 \mathrm{c}$ & $0.40 \mathrm{c}$ & $0.74 \mathrm{c}$ & $0.49 \mathrm{c}$ & $0.88 \mathrm{c}$ & $0.73 \mathrm{c}$ & $2.39 \mathrm{c}$ & $1.31 \mathrm{c}$ & $2.63 \mathrm{c}$ & $1.34 \mathrm{c}$ & $3.44 \mathrm{c}$ & $2.07 \mathrm{c}$ \\
\hline 60 & $0.86 \mathrm{~b}$ & $0.64 \mathrm{~b}$ & $1.16 \mathrm{~b}$ & $0.76 \mathrm{~b}$ & $1.34 \mathrm{~b}$ & $1.17 \mathrm{~b}$ & $3.39 \mathrm{~b}$ & $2.02 \mathrm{~b}$ & $3.84 \mathrm{~b}$ & $1.92 \mathrm{~b}$ & $4.60 \mathrm{~b}$ & $2.98 \mathrm{~b}$ \\
\hline 120 & $1.15 \mathrm{a}$ & $0.89 \mathrm{a}$ & $1.77 \mathrm{a}$ & $1.07 \mathrm{a}$ & $2.10 \mathrm{a}$ & $1.76 \mathrm{a}$ & $4.52 \mathrm{a}$ & $2.75 \mathrm{a}$ & $5.53 \mathrm{a}$ & $2.52 \mathrm{a}$ & $6.52 \mathrm{a}$ & $4.24 \mathrm{a}$ \\
\hline LSD & 0.054 & 0.038 & 0.087 & 0.034 & 0.117 & 0.100 & 0.148 & 0.102 & 0.271 & 0.113 & 0.260 & 0.217 \\
\hline $\mathbf{C} \times \mathbf{S}$ & $<0.015^{*}$ & $<0.001^{*}$ & $<0.004 *$ & $<0.001 *$ & $0.116 \mathrm{~ns}$ & $0.062 \mathrm{~ns}$ & $0.075 \mathrm{~ns}$ & $<0.001 *$ & $0.169 \mathrm{~ns}$ & $<0.007 *$ & $0.051 \mathrm{~ns}$ & $<0.047 *$ \\
\hline $\mathbf{C} \times \mathbf{N}$ & $<0.002 *$ & $<0.001^{*}$ & $<0.001 *$ & $<0.001 *$ & $<0.001 *$ & $<0.001 *$ & $<0.003 *$ & $<0.001 *$ & $<0.001^{*}$ & $<0.001 *$ & $<0.001 *$ & $<0.001 *$ \\
\hline $\mathbf{S} \times \mathbf{N}$ & $<0.022 *$ & $<0.001^{*}$ & $<0.002 *$ & $<0.001 *$ & $0.681 \mathrm{~ns}$ & $<0.028^{*}$ & $<0.023^{*}$ & $<0.001 *$ & $<0.004^{*}$ & $0.941 \mathrm{~ns}$ & $0.353 \mathrm{~ns}$ & $0.059 \mathrm{~ns}$ \\
\hline $\mathbf{C} \times \mathbf{S} \times \mathbf{N}$ & $0.502 \mathrm{~ns}$ & $0.192 \mathrm{~ns}$ & $0.713 \mathrm{~ns}$ & $0.258 \mathrm{~ns}$ & $0.889 \mathrm{~ns}$ & $0.066 \mathrm{~ns}$ & $0.400 \mathrm{~ns}$ & $0.129 \mathrm{~ns}$ & $0.673 \mathrm{~ns}$ & $0.172 \mathrm{~ns}$ & $0.996 \mathrm{~ns}$ & $0.088 \mathrm{~ns}$ \\
\hline C.V. (\%) & 11.3 & 10.7 & 12.9 & 8.1 & 14.7 & 14.9 & 7.8 & 9.1 & 12.3 & 10.6 & 9.7 & 12.7 \\
\hline
\end{tabular}

ns, non-significant; *, significant at $P \leq 0.05 ; * *, P \leq 0.0$

Different letters indicate statistically significant-difference among the values in each column and individual factors (SNK test; $P \leq 0.05$ ) 
Table 3. Interactive effect of Cultivar, Seed rate and Nitrogen application of sorghum forage at different growth stages during 2008 and 2009

\begin{tabular}{|c|c|c|c|c|c|c|c|c|c|c|c|c|}
\hline \multirow{3}{*}{ Treatments } & \multicolumn{6}{|c|}{ Ether extractable fat (\%) } & \multicolumn{6}{|c|}{ Total ash content $(\%)$} \\
\hline & \multicolumn{2}{|c|}{ Pre-booting } & \multicolumn{2}{|c|}{ Booting } & \multicolumn{2}{|c|}{$50 \%$ heading } & \multicolumn{2}{|c|}{ Pre-booting } & \multicolumn{2}{|c|}{ Booting } & \multicolumn{2}{|c|}{$50 \%$ heading } \\
\hline & 2008 & 2009 & 2008 & 2009 & 2008 & 2009 & 2008 & 2009 & 2008 & 2009 & 2008 & 2009 \\
\hline \multicolumn{13}{|l|}{ Cultivar (C) } \\
\hline JS-2002 & $3.0 \mathrm{a}$ & $1.9 \mathrm{a}$ & $2.8 \mathrm{a}$ & $1.7 \mathrm{a}$ & $2.6 \mathrm{a}$ & $1.5 \mathrm{a}$ & $8.1 \mathrm{a}$ & $7.4 \mathrm{a}$ & $8.5 \mathrm{a}$ & $7.9 \mathrm{a}$ & $9.5 \mathrm{a}$ & $8.6 \mathrm{a}$ \\
\hline Chakwal sorghum & $2.7 \mathrm{~b}$ & $1.7 \mathrm{~b}$ & $2.6 \mathrm{~b}$ & $1.5 \mathrm{~b}$ & $2.4 \mathrm{~b}$ & $1.3 \mathrm{~b}$ & $7.6 \mathrm{~b}$ & $6.7 \mathrm{~b}$ & $8.0 \mathrm{~b}$ & $7.4 \mathrm{~b}$ & $8.8 \mathrm{~b}$ & $7.9 \mathrm{~b}$ \\
\hline JS-263 & $2.2 \mathrm{c}$ & $1.1 \mathrm{c}$ & $2.1 \mathrm{c}$ & $1.0 \mathrm{c}$ & $1.9 \mathrm{c}$ & $0.83 \mathrm{c}$ & $6.7 \mathrm{c}$ & $6.0 \mathrm{c}$ & $7.3 \mathrm{c}$ & $6.7 \mathrm{c}$ & $7.9 \mathrm{c}$ & $7.2 \mathrm{c}$ \\
\hline LSD & 0.194 & 0.065 & 0.131 & 0.079 & 0.141 & 0.075 & 0.311 & 0.339 & 0.378 & 0.265 & 0.363 & 0.238 \\
\hline \multicolumn{13}{|c|}{ Seed rate $(S)\left(\mathrm{kg} \mathrm{ha}^{-1}\right)$} \\
\hline 75 & $3.0 \mathrm{a}$ & $1.9 \mathrm{a}$ & $2.7 \mathrm{a}$ & $1.7 \mathrm{a}$ & $2.6 \mathrm{a}$ & $1.4 \mathrm{a}$ & $6.8 \mathrm{c}$ & $6.1 \mathrm{c}$ & $7.5 \mathrm{c}$ & $7.0 \mathrm{c}$ & $8.2 \mathrm{c}$ & $7.3 \mathrm{c}$ \\
\hline 100 & $2.6 \mathrm{~b}$ & $1.5 \mathrm{~b}$ & $2.4 \mathrm{~b}$ & $1.4 \mathrm{~b}$ & $2.3 \mathrm{~b}$ & $1.2 \mathrm{~b}$ & $7.5 \mathrm{~b}$ & $6.7 \mathrm{~b}$ & $8.0 \mathrm{~b}$ & $7.4 \mathrm{~b}$ & $8.7 \mathrm{~b}$ & $7.9 \mathrm{~b}$ \\
\hline 125 & $2.4 \mathrm{c}$ & $1.3 \mathrm{c}$ & $2.2 \mathrm{c}$ & $1.2 \mathrm{c}$ & $2.1 \mathrm{c}$ & $1.0 \mathrm{c}$ & $8.0 \mathrm{a}$ & $7.3 \mathrm{a}$ & $8.3 \mathrm{a}$ & $7.6 \mathrm{a}$ & $9.3 \mathrm{a}$ & $8.5 \mathrm{a}$ \\
\hline LSD & 0.194 & 0.065 & 0.131 & 0.079 & 0.141 & 0.075 & 0.311 & 0.339 & 0.378 & 0.265 & 0.363 & 0.238 \\
\hline \multicolumn{13}{|c|}{ Nitrogen level (N) $\left(\mathrm{kg} \mathrm{ha}^{-1}\right)$} \\
\hline 0 & $2.2 \mathrm{c}$ & $1.1 \mathrm{c}$ & $2.0 \mathrm{c}$ & $0.99 \mathrm{c}$ & $1.9 \mathrm{c}$ & $0.84 \mathrm{c}$ & $6.7 \mathrm{c}$ & $5.9 \mathrm{c}$ & $7.2 \mathrm{c}$ & $6.4 \mathrm{c}$ & $8.0 \mathrm{c}$ & $7.0 \mathrm{c}$ \\
\hline 60 & $2.8 \mathrm{~b}$ & $1.7 \mathrm{~b}$ & $2.6 \mathrm{~b}$ & $1.5 \mathrm{~b}$ & $2.4 \mathrm{~b}$ & $1.3 \mathrm{~b}$ & $7.6 \mathrm{~b}$ & $6.8 \mathrm{~b}$ & $7.9 \mathrm{~b}$ & $7.3 \mathrm{~b}$ & $8.8 \mathrm{~b}$ & $8.1 \mathrm{~b}$ \\
\hline 120 & $3.0 \mathrm{a}$ & $1.9 \mathrm{a}$ & $2.8 \mathrm{a}$ & $1.7 \mathrm{a}$ & $2.7 \mathrm{a}$ & $1.5 \mathrm{a}$ & $8.1 \mathrm{a}$ & $7.4 \mathrm{a}$ & $8.7 \mathrm{a}$ & $8.2 \mathrm{a}$ & $9.5 \mathrm{a}$ & $8.6 \mathrm{a}$ \\
\hline LSD & 0.194 & 0.065 & 0.131 & 0.079 & 0.141 & 0.075 & 0.311 & 0.339 & 0.378 & 0.265 & 0.363 & 0.238 \\
\hline $\mathbf{C} \times \mathbf{S}$ & $0.944 \mathrm{~ns}$ & $0.645 \mathrm{~ns}$ & $0.999 \mathrm{~ns}$ & $0.226 \mathrm{~ns}$ & $0.782 \mathrm{~ns}$ & $0.578 \mathrm{~ns}$ & $<0.006^{*}$ & $0.188 \mathrm{~ns}$ & $0.679 \mathrm{~ns}$ & $0.996 \mathrm{~ns}$ & $0.862 \mathrm{~ns}$ & $0.852 \mathrm{~ns}$ \\
\hline $\mathbf{C} \times \mathbf{N}$ & $0.558 \mathrm{~ns}$ & $<0.001 * *$ & $0.104 \mathrm{~ns}$ & $<0.001 * *$ & $<0.036^{*}$ & $<0.001 * *$ & $0.729 \mathrm{~ns}$ & $0.797 \mathrm{~ns}$ & $0.540 \mathrm{~ns}$ & $<0.015^{*}$ & $0.956 \mathrm{~ns}$ & $0.205 \mathrm{~ns}$ \\
\hline $\mathbf{S} \times \mathbf{N}$ & $0.722 \mathrm{~ns}$ & $<0.003 *$ & $0.863 \mathrm{~ns}$ & $0.284 \mathrm{~ns}$ & $0.852 \mathrm{~ns}$ & $0.363 \mathrm{~ns}$ & $0.921 \mathrm{~ns}$ & $0.913 \mathrm{~ns}$ & $0.984 \mathrm{~ns}$ & $0.965 \mathrm{~ns}$ & $0.641 \mathrm{~ns}$ & $<0.016^{*}$ \\
\hline $\mathbf{C} \times \mathbf{S} \times \mathbf{N}$ & $0.989 \mathrm{~ns}$ & $0.183 \mathrm{~ns}$ & $0.973 \mathrm{~ns}$ & $0.941 \mathrm{~ns}$ & $0.961 \mathrm{~ns}$ & $0.622 \mathrm{~ns}$ & $0.975 \mathrm{~ns}$ & $0.623 \mathrm{~ns}$ & $0.999 \mathrm{~ns}$ & $0.983 \mathrm{~ns}$ & $0.975 \mathrm{~ns}$ & $0.815 \mathrm{~ns}$ \\
\hline C.V. (\%) & 13.3 & 7.6 & 9.7 & 10.2 & 11.0 & 11.3 & 7.6 & 9.2 & 8.6 & 6.6 & 7.5 & 5.5 \\
\hline
\end{tabular}

Different letters indicate statistically significant-difference among the values in each column and individual factors (SNK test; $P \leq 0.05$ ) 
Table 4. Interactive effect of Cultivar, Seed rate and Nitrogen application of sorghum forage at different growth stages during 2008 and 2009

\begin{tabular}{|c|c|c|c|c|c|c|c|c|c|c|c|c|}
\hline \multirow{3}{*}{ Treatments } & \multicolumn{6}{|c|}{ Nitrogen free extract (\%) } & \multicolumn{6}{|c|}{ Organic matter (\%) } \\
\hline & \multicolumn{2}{|c|}{ Pre-booting } & \multicolumn{2}{|c|}{ Booting } & \multicolumn{2}{|c|}{$50 \%$ heading } & \multicolumn{2}{|c|}{ Pre-booting } & \multicolumn{2}{|c|}{ Booting } & \multicolumn{2}{|c|}{$50 \%$ heading } \\
\hline & 2008 & 2009 & 2008 & 2009 & 2008 & 2009 & 2008 & 2009 & 2008 & 2009 & 2008 & 2009 \\
\hline \multicolumn{13}{|l|}{ Cultivar (C) } \\
\hline JS-2002 & $35.6 \mathrm{c}$ & $38.3 \mathrm{c}$ & $47.3 \mathrm{c}$ & $49.0 \mathrm{c}$ & $51.4 \mathrm{c}$ & $53.4 \mathrm{c}$ & 23.9 a & $17.1 \mathrm{a}$ & 27.4 & $19.1 \mathrm{a}$ & 34.4 & $29.1 \mathrm{a}$ \\
\hline $\begin{array}{l}\text { Chakwal } \\
\text { sorghum }\end{array}$ & $41.8 \mathrm{~b}$ & $43.4 \mathrm{~b}$ & $52.3 \mathrm{~b}$ & $54.2 \mathrm{~b}$ & $54.2 \mathrm{~b}$ & $56.5 \mathrm{~b}$ & $16.8 \mathrm{~b}$ & $12.7 \mathrm{~b}$ & 27.2 & $15.0 \mathrm{~b}$ & 31.5 & $26.3 \mathrm{~b}$ \\
\hline JS-263 & $47.7 \mathrm{a}$ & $49.4 \mathrm{a}$ & $57.4 \mathrm{a}$ & $59.5 \mathrm{a}$ & $58.2 \mathrm{a}$ & $60.4 \mathrm{a}$ & $15.3 \mathrm{c}$ & $10.2 \mathrm{c}$ & 23.6 & $12.1 \mathrm{c}$ & 32.0 & $27.0 \mathrm{~b}$ \\
\hline LSD & 1.050 & 0.712 & 1.126 & 1.386 & 0.831 & 0.892 & 0.811 & 0.525 & $0.067 \mathrm{~ns}$ & 0.623 & $0.133 \mathrm{~ns}$ & 0.763 \\
\hline \multicolumn{13}{|c|}{ Seed rate $(S)\left(\mathrm{kg} \mathrm{ha}^{-1}\right)$} \\
\hline 75 & $38.7 \mathrm{c}$ & $41.0 \mathrm{c}$ & $49.6 \mathrm{c}$ & $51.6 \mathrm{c}$ & $52.0 \mathrm{c}$ & $54.2 \mathrm{c}$ & $16.4 \mathrm{c}$ & $11.5 \mathrm{c}$ & $23.8 \mathrm{c}$ & $14.2 \mathrm{c}$ & $30.6 \mathrm{c}$ & $24.5 \mathrm{c}$ \\
\hline 100 & $41.4 \mathrm{~b}$ & $43.5 \mathrm{~b}$ & $52.1 \mathrm{~b}$ & $54.1 \mathrm{~b}$ & $54.7 \mathrm{~b}$ & $56.9 \mathrm{~b}$ & $18.6 \mathrm{~b}$ & $13.3 \mathrm{~b}$ & $25.9 \mathrm{~b}$ & $14.9 \mathrm{~b}$ & $31.8 \mathrm{~b}$ & $26.6 \mathrm{~b}$ \\
\hline 125 & $44.9 \mathrm{a}$ & $46.4 \mathrm{a}$ & $55.1 \mathrm{a}$ & $57.1 \mathrm{a}$ & $57.2 \mathrm{a}$ & $59.2 \mathrm{a}$ & $21.0 \mathrm{a}$ & $15.3 \mathrm{a}$ & $28.4 \mathrm{a}$ & $17.2 \mathrm{a}$ & $35.4 \mathrm{a}$ & $31.3 \mathrm{a}$ \\
\hline LSD & 1.050 & 0.712 & 1.126 & 1.386 & 0.831 & 0.892 & 0.811 & 0.525 & 0.960 & 0.623 & 0.934 & 0.763 \\
\hline \multicolumn{13}{|c|}{ Nitrogen level (N) $\left(\mathrm{kg} \mathrm{ha}^{-1}\right)$} \\
\hline 0 & $46.1 \mathrm{a}$ & $48.6 \mathrm{a}$ & $56.0 \mathrm{a}$ & $58.3 \mathrm{a}$ & $58.6 \mathrm{a}$ & $60.4 \mathrm{a}$ & $16.3 \mathrm{c}$ & $11.2 \mathrm{c}$ & $23.2 \mathrm{c}$ & $13.2 \mathrm{c}$ & $30.4 \mathrm{c}$ & $25.5 \mathrm{c}$ \\
\hline 60 & $41.3 \mathrm{~b}$ & $43.2 \mathrm{~b}$ & $52.2 \mathrm{~b}$ & $54.2 \mathrm{~b}$ & $54.7 \mathrm{~b}$ & $56.7 \mathrm{~b}$ & $18.5 \mathrm{~b}$ & $13.6 \mathrm{~b}$ & $26.2 \mathrm{~b}$ & $15.6 \mathrm{~b}$ & $32.4 \mathrm{~b}$ & $26.9 \mathrm{~b}$ \\
\hline 120 & $37.7 \mathrm{c}$ & $39.2 \mathrm{c}$ & $48.7 \mathrm{c}$ & $50.1 \mathrm{c}$ & $50.6 \mathrm{c}$ & $53.2 \mathrm{c}$ & $21.3 \mathrm{a}$ & $15.4 \mathrm{a}$ & $28.8 \mathrm{a}$ & $17.4 \mathrm{a}$ & $35.0 \mathrm{a}$ & $30.0 \mathrm{a}$ \\
\hline LSD & 1.050 & 0.712 & 1.126 & 1.386 & 0.831 & 0.892 & 0.811 & 0.525 & 0.960 & 0.623 & 0.934 & 0.763 \\
\hline $\mathbf{C} \times \mathbf{S}$ & $0.143 \mathrm{~ns}$ & $<0.038^{*}$ & $0.647 \mathrm{~ns}$ & $0.585 \mathrm{~ns}$ & $0.589 \mathrm{~ns}$ & $0.811 \mathrm{~ns}$ & $<0.009 *$ & $<0.001 * *$ & $0.467 \mathrm{~ns}$ & $<0.008^{*}$ & $0.503 \mathrm{~ns}$ & $<0.001 * *$ \\
\hline $\mathbf{C} \times \mathbf{N}$ & $0.997 \mathrm{~ns}$ & $0.434 \mathrm{~ns}$ & $0.517 \mathrm{~ns}$ & $0.549 \mathrm{~ns}$ & $0.871 \mathrm{~ns}$ & $0.557 \mathrm{~ns}$ & $0.483 \mathrm{~ns}$ & $0.203 \mathrm{~ns}$ & $0.400 \mathrm{~ns}$ & $<0.033 *$ & $<0.001 * *$ & $<0.001 * *$ \\
\hline $\mathbf{S} \times \mathbf{N}$ & $0.989 \mathrm{~ns}$ & $<0.036^{*}$ & $0.874 \mathrm{~ns}$ & $0.904 \mathrm{~ns}$ & $0.540 \mathrm{~ns}$ & $0.087 \mathrm{~ns}$ & $0.233 \mathrm{~ns}$ & $<0.001 * *$ & $0.489 \mathrm{~ns}$ & $<0.023^{*}$ & $0.341 \mathrm{~ns}$ & $0.088 \mathrm{~ns}$ \\
\hline $\mathbf{C} \times \mathbf{S} \times \mathbf{N}$ & $0.810 \mathrm{~ns}$ & $<0.006^{*}$ & $0.999 \mathrm{~ns}$ & $0.541 \mathrm{~ns}$ & $0.873 \mathrm{~ns}$ & $0.656 \mathrm{~ns}$ & $0.186 \mathrm{~ns}$ & $<0.004 * *$ & $0.278 \mathrm{~ns}$ & $<0.006^{*}$ & $0.844 \mathrm{~ns}$ & $0.070 \mathrm{~ns}$ \\
\hline C.V. (\%) & 4.6 & 3.0 & 3.9 & 4.6 & 2.6 & 2.8 & 7.9 & 7.1 & 6.5 & 7.3 & 5.2 & 5.0 \\
\hline
\end{tabular}

ns, non-significant; *, significant at $P \leq 0.05 ; * *, P \leq 0.01$

Different letters indicate statistically significant-difference among the values in each column and individual factors (SNK test; $P \leq 0.05$ ) 


\section{DISCUSSION}

The nutritive value of forage is a measure of proximate composition, digestibility and nature of digested products and thereby its ability to maintain or promote growth, milk production or other physiological function in the animal body. The forage digestibility is related to change in chemical composition and to some extent crude protein (Sher et al., 2014; Bose and Balakarishan, 2001). Therefore; forage containing high crude protein content is considered of good quality. Current study indicates that forage sorghum cultivars influenced the proximate compositions at different developmental stages. Cultivar JS-2002 appeared to be more nutritious for animals as it contains high protein in comparison with other two cultivars, it might be due to its genetic potential (Celen and Akdemir, 1998). Shobha et al. (2008) found that crude protein between 8 and $13 \%$ of sorghum genotypes that was in line with present study having the crude protein 7 13\%. Aruna et al. (2015), Rana et al. (2014), Sarfraz et al. (2012), Ayub et al. (2010), Abusuwar and Hala (2010), Mahammed and Moataz (2009) and Carmi et al. (2006) have also reported significant differences among the sorghum cultivars for crude protein. Likewise; Ayub et al. (2012) found the variation in protein contents among the various sorghum cultivars. In contrast Eltelib and Eltom (2006) and Mahmud et al. (2003) did not find any significant differences in protein contents among various sorghum cultivars.

However, increasing the seed rates decreased crude protein of sorghum forage. Similar results were found by Ayub et al. (2003) who examined that crude protein decreased by increasing seed rate, while Iptas et al. (2002) found contradictory results that no statistically significant association was observed in seed rate and crude protein.

The current studies showed that application of $\mathrm{N}$ significantly enhanced the crude protein in sorghum forage, which might be due to high concentration of amino acids, because $\mathrm{N}$ directly involved in synthesis of amino acid (Sher et al., 2016). Our results are supported by many researchers who reported that crude protein content increased with increasing $\mathrm{N}$ levels (Glamoclija et al., 2011; Almodares et al., 2009; Iptas and Brohi 2003; Iptas et al., 2002). Crude protein was found $8-13 \%$ at NP fertilizer, $7-9 \%$ at $\mathrm{N}-\mathrm{K}_{2} \mathrm{O}$ rates, $4-8 \%$ and $5-8 \%$ at NPK fertilizer (Mahmud et al., 2003; Pholsen and Sornsungnoen, 2004; Pholsen and Sukrsi, 2004; Azam et al., 2010) whereas in our experiment 6-13\% was recorded which is similar to previous investigations. Protein is one of the most costly supplements for livestock; the total amount of protein produced per unit area is one of the most important quality characteristics as suggested by many researchers (Assefa and Ledin, 2001; Lithourgidis et al., 2006; Atis et al., 2012). The result of the current study was in line with finding of Kim et al. (2006) and Bhilare et al. (2007) who also reported that application of $\mathrm{N}$ increased the crude protein yield while some studies showed the contradictory results (Iqbal et al., 2013; Iptas and Brohi 2003) who reported that no significant differences were found by the application of $\mathrm{N}$ rate.
The intake, palatability and digestibility of forage is inversely related to crude fibre percentage i.e. the forage containing low crude fibre content is better in quality and vice versa. Therefore, in present investigations, cultivar JS-2002 produced low contents of crude fibre in comparison with other two cultivars that might be due to the genetic makeup the of the cultivars. Rana et al. (2014), Abusuwar and Hala (2010), Ayub et al. (2010; 2012), Shobha et al. (2008), Nabi et al. (2006) also supported our finding and confirmed that there was a significant difference among sorghum varieties regarding crude fibre. In case of seed rate, it was found that crude fibre contents were decreasing with increasing the seed rate. Almost similar results have been reported by Ayub et al. (2003) who also found that crude fibre decreased with the increasing of seed rate while Abusuwar (1994) found the contradictory results. Good quality forage has less crude fibre and in case of higher crude fibre percentage digestibility will be reduced. Increased $\mathrm{N}$ levels increased the crude fibre (Eltelib and Eltom, 2006; Mahmud et al., 2003) whereas current study revealed that higher crude fibre was recorded with the application of NP while Almodares et al. (2009) found the contradictory results with current study.

In current study, there is variation among the cultivars of sorghum for EE. This might be due to their genetic characters. Ayub et al. (2002, 2010), Mahammed and Moataz (2009) and Shobha et al. (2008) also found the significant difference in $\mathrm{EE}$ of various cultivars. In addition; concentration of $\mathrm{EE}$ is increased with increasing the dose of $\mathrm{N}$ which might be due to higher production of glycerol contents which lead to an increased fats percentage. According to our findings, results were consistent with the results of Azam et al. (2010), Ayub et al. (2002) who reported that EE increases with an increase in $\mathrm{N}$ levels.

In our results it was observed that ash percentage varied among the sorghum cultivars which might be due to genetic potential and rooting array to absorb nutrients from soil (Rana et al. 2014; Ayub et al. 2012). An increasing trend for total ash content was observed and our results are consistent with the results of Ayub et al. (2003) who found that total ash content increased with increasing seed rate. Similarly, nitrogen has direct relationship with total ash content in present study. Ayub et al. (2002) found that increased level of fertilizer increased the total ash content. Mahmud et al. (2003) observed maximum total ash content at the highest rate of NP fertilizer levels (100-100 $\left.\mathrm{kg} \mathrm{ha}^{-1}\right)$, whereas in our experiment the maximum total ash content was recorded with the application of NP (120-30 $\left.\mathrm{kg} \mathrm{ha}^{-1}\right)$.

Glamoclija et al. (2011) reported significant differences for NFE in different cultivars. However, in our experiment sorghum cultivar JS-263 seemed well adapted under local climate and utilized the available resources efficiently as compared to other two cultivars. The $\mathrm{N}$ rate has inversely proportional for NFE. Similar results are reported by Rakic et al. (2013) and Glamoclija et al. (2011) who found that NFE decreased with increasing $\mathrm{N}$ 
levels. Our results presents that highest OM was observed from JS-2002 compared to JS-263. Increased seed and N rates gradually increased the OM. It was higher in 2009 than 2008 indicating some sessional effects on cultivars.

\section{CONCLUSION}

The JS-2002 indicate more efficiency in CP, CPY, CF, CFY, TA, EE, OM while JS-263 in NFE than Chakwal sorghum. The proximate composition would be affected by the variation of seed rate at the given developmental stages. In this study, high fodder quality was expressed by the forage sorghum cultivars JS-2002 at $75 \mathrm{~kg} \mathrm{ha}^{-1}$ seed rate and $\mathrm{N}$ fertilization of $120 \mathrm{~kg} \mathrm{ha}^{-1}$. Moreover; these treatments are suitable to harvest at $50 \%$ heading stage and recommended best for livestock feeding.

\section{LITERATURE CITED}

Abusuwar, A.O. 1994. Effect of seeding rates, sowing methods and fertilizer application on the performance of fodder sorghum grown on a saline-sodic soil. Uni. Khartoum J. Agric. Sci. 2(1): 28-45

Abusuwar, A.O. and Hala. 2010. Effect of chicken manure on yield, quality and $\mathrm{HCN}$ concentration of two forage sorghum (Sorghum bicolor (L.) Moench) cultivars. Agric. Biol. J. North Amer. 1: 27-31.

Almodares, A., M. Jafarinia and M.R. Hadi. 2009. The effects of nitrogen fertilizer on chemical compositions in corn and sweet sorghum. American-Eurasian J. Agric. Environ. Sci. 6(4): 441-446.

AOAC, 2002. Official Method of Analysis, Association of Official Analytical Chemist, $17^{\text {th }}$ Ed. Arlington, Virginia USA.

Aruna, C., M. Swarnalatha, P.P. Kumar, V. Devender, M. Suguna, M. Blümmel and J.V. Patil. 2015. Genetic options for improving fodder yield and quality in forage sorghum. Tropical Grasslands - Forrajes Tropicales, 3: 49-58.

Ashraf, Y., S.A. Nagre and A.H. Gillani. 1995. Micro mineral contents of fodder as affected by varieties and harvesting intervals. J. Agric. Res. 33: 421-425.

Assefa, G. and I. Ledin. 2001. Effect of variety, soil type and fertilizer on the establishment, growth, forage yield, quality and voluntary intake by cattle of oats and vetches cultivated in pure stands and mixtures. Anim. Feed Sci. Technol. 92: 95-111.

Ayub, M., T. Asif, A. Safdar and M. Nadeem. 2002. Effect of different nitrogen levels and seed rates on growth, yield and quality of sorghum (Sorghum bicolor) fodder. Ind. J. Agric. Sci. 72(11): 648-650.

Ayub, M., M. Khalid, M. Tariq, M. Elahi and M.A. Nadeem. 2012. Comparison of sorghum genotypes for forage production and quality. J. Ani. Pl. Sci. 22: 733-737.

Ayub, M., M.A. Nadeem, M. Tahir, A. Ghafoor, Z. Ahmed and M. Naeem. 2010. Comparative studies on the growth, forage yield and quality of sorghum (Sorghum bicolor L.) varieties under irrigated conditions of Faisalabad. Pak. J. Life Soc. Sci. 8: 94-97.

Ayub, M., A. Tanveer, M.A. Nadeem and M. Tayyub. 2003. Fodder yield and quality of sorghum (Sorghum bicolor L.) as influenced by different tillage methods and seed rates. Pak. J. Agron. 2(3): 179-184.

Azam, M., E.A. Waraich, A. Pervaiz and F. Nawaz. 2010. Response of a newly developed fodder sorghum (Bicolor $\mathrm{L}$. Monech) variety (F-9917) to NPK application. Pak. J. Life Soc. Sci. 8(2): 117-120.
Bhilare, R.L. and Y.P. Joshi. 2007. Productivity and quality of oat (Avena sative $\mathrm{L}$.) in relation to cutting management and nitrogen levels. Ind. J. Agron. 52(3): 247-250.

Bilal, M.Q., M. Abdullah and M. Lateef. 2001. Effect of mott dwarf elephant grass (Pennisetum purpureum) silage on dry matter intake, milk production, digestibility and rumen characteristics in Nili-Ravi buffaloes. In: Proc. 54th Annua Reciprocal Meat Conference (Vol. II). Indianapolis Indiana, USA.

Bose, M.S.C. and V. Balakrishnan. 2001. Forage production technologies. South Asian Publication Pvt. Ltd. New Delhi, India. 253pp.

Carmi, A., Y. Aharoni, M. Edelstein, N. Umiel, A. Hagiladi, E. Yosef, M. Nikbachat and J. Miron. 2006. Effects of irrigation and plant density on yield, composition and in vitro digestibility of a new forage sorghum variety, Tal, at two maturity stages. J. Anim. Sci. 131:120-132.

Çelen, A.E. and H. Akdemir. 1998. Effects of cutting time and nitrogen fertilization on forage yield and quality of a sorghum-Sudangrass hybrid. Turk. J. Field Crops 3(1): 2529.

Chohan, M.S.M., M. Naeem, A.H. Khan and R.A. Kainth. 2006. Performance of pearl millet (Pennisetum americanumL.) varieties for forage yield. J. Agric. Res. 44: 23-27.

Clipson, N.W. and S.J. Edwards. 1994. Factors affecting crop productivity; the biotic environment. In crop productivity (Eds. Weston G.D), Botter worth, Heinenemann ltd. Jordan hall, oxford, UK.

Eltelib, H.A. and E.A. Eltom. 2006. Effect of time of nitrogen application on growth, yield and quality of four forage sorghum cultivars. Agric. J. 1(2): 59-63.

Glamoclija, D., S. Jankovic, S. Rakic, R. Maletic, J. Ikanovic and Z. Lakic. 2011. Effects of nitrogen and harvesting time on chemical composition of biomass of Sudan grass, fodder sorghum, and their hybrid. Turk. J. Agric. 35: 1-12.

Iptas, S. and A.R. Brohi. 2003. Effect of nitrogen rate and stubble height on dry matter yield, crude protein content and crude protein yield of a sorghum-sudangrass hybrid [Sorghum bicolor (L.) Moench $\times$ sorghum sudanense (Piper) Stapf] in the three-cutting system. J. Agron. Crop Sci. 189: 227-232.

Iptas, S., A.R. Brohi and H. Aslan. 2002. Effect of seeding rate and nitrogen fertilizers on forage yield and quality of sorghum (Sorghum bicolor) $\mathrm{x}$ sudangrass (Sorghum sudanense) hybrid. Ind. J. Agron. 47(2): 298-303.

Iqbal, M.F., Z. Iqbal, M. Farooq, L. Ali and M. Fiaz. 2013. Impact nitrogenous fertilizer on yield and quality of oat. Pak. J. Sci. 65(1): 1-4.

Kim, D.J., S.G. Kim, S.J. Abuel, C.H. Kwon, C.N. Shin, K.H. Ko and B.G. Park. 2006. Effect of location, season and variety on yield and quality of forage oat. Asian-Aust. J. Anim. Sci. 19(7): 970-977.

Kumar, A. and K.S. Thakur. 2006. Effect of Brassica spp., and their sowing proportions on productivity, competition and economics of wheat (Triticum aestivum) + Brassica mixed cropping system under rainfed conditions of Himachal Pradesh. Ind. J. Agron. 51: 259-262.

Lithourgidis, A.S., I.B. Vasilakoglou, K.V. Dhima, C.A. Dordas and M.D. Yiakoulaki. 2006. Forage yield and quality of common vetch mixtures with oat and triticale in two seeding ratios. Field Crops Res. 99: 106-113.

Mahmud, K., I. Ahmad and M. Ayub. 2003. Effect of nitrogen and phosphorus on the fodder yield and quality of two sorghum variety (Sorghum bicolor L.). Int. J. Agri. Biol. 5: 61-63.

Mohammed, A.A.H. and Y.N. Hameed. 1988. The effect of cutting stage, nitrogen fertilization and seeding rate on yield 
and quality of hybrid forage sorghum. Iraqi J. Agric. Sci. Zanco, 6: 125-138.

Mohammed, M.I. and M.A. Moataz. 2009. Evaluation of newly developed sweet sorghum (Sorghum bicolor) genotypes for some forage attributes. American-Eurasian J. Agric. Environ. Sci. 6(4): 434-440.

Nabi, C.G., M. Riaz and G. Ahmad. 2006. Comparison of some advanced lines of Sorghum bicolor L. Monech for green fodder/dry matter yields and morpho-economic parameters. J. Agric. Res. 44: 191-198.

Patel, G.N., P.G. Patel and J.C. Patel. 1994. Effect of nitrogen and phosphorus on yield and quality of forage sorghum (sorghum bicolor L.). Ind. J. Agron. 39: 123-125.

Pholsen, S. And N. Somsungnoen. 2004. Effect of nitrogen and potassium rates and planting distances on growth, yield and fodder quality of a forage sorghum (Sorghum bicolor L. Moench). Pak. J. Bio. Sci. 7(10): 1793-1800.

Pholsen, S. and A. Suksri. 2004. Effect of organic amendment and chemical fertilizer on growth, yield and fodder quality of a forage sorghum (Sorghum bicolor L. Moench). Pak. J. Bio. Sci. 7(4): 651-657.
Rakić, S., Đ. Glamočlija, J. Ikanović, S. Janković and M. Živković. 2013. Morphological traits, yield and chemical composition of forage sorghum genotypes, grown under different nitrogen rates. Romanian Agric. Res. 30: 109-115.

Rana, A.S., A.H. Ahmad, N. Saleem, A. Nawaz, T. Hussian and M. Saad. 2014. Differential response of sorghum cultivars for fodder yield and quality. J. Glob. Innov. Agric. Soc. Sci. 2(1): 6-10.

Sarfraz, M., N. Ahmad, U. Farooq, A. Ali and K. Hussain. 2012. Evaluation of sorghum varieties/lines for Hydrocyanic acid and crude protein contents. J. Agric. Res. 50(1): 39-47.

Sher, A., M. Ansar, A. Manaf, A. Qayyum, M. F. Saeed and M. Irfan. 2014. Hydrocyanic acid and sugar content dynamics under nitrogen and sulphur application to forage sorghum cultivars. Turk. J. Field Crops 19(1): 46-52.

Sher, A., F.U. Hassan, H. Ali and W. Hassan. 2016. Seed rate and nitrogen application effects on production and brix value of forage sorghum cultivars. Grassland Sci. 62: 119-127.

Shobha, V., B. Kasturiba, R.K. Naik and N. Yenagi. 2008. Nutritive value and quality characteristics of sorghum genotypes. Karnataka J. Agric. Sci. 20: 586-588. 